Classification

Physics Abstracts

$67.80-64.70 \mathrm{D}-81.10 \mathrm{~F}$

\title{
Liquid-solid interface inertia
}

\author{
L. Puech and B. Castaing \\ Centre de Recherches sur les Très Basses Températures, \\ C.N.R.S., BP 166X, 38042 Grenoble Cedex, France
}

(Reçu le 22 février 1982, révisé le 13 avril, accepté le 28 juin 1982)

\begin{abstract}
Résumé. - Nous remarquons que, lors de la fusion ou de la croissance d'un cristal, les atomes se réarrangent à l'interface à cause de la différence de structure entre liquide et solide. Il en résulte une énergie " cinétique " de surface. Nous étudions les conséquences de ce terme dans le cas de ${ }^{4} \mathrm{He}$ où l'interface est très mobile. La relation de dispersion des ondes de cristallisation devient linéaire : $\omega=c_{0} k$. La transmission, à travers l'interface, des phonons est augmentée. La résistance de Kapitza est donc diminuée, mais presente toujours le comportement anormal $R_{\mathrm{K}} \propto T^{-5}$ prédit par Marchenko et Parshin sur d'autres bases.
\end{abstract}

\begin{abstract}
We remark that, during crystallization or melting, due to the structural difference between solid and liquid, the atoms have to rearrange themselves at the interface. This results in a supplementary surface " kinetic" energy. We examine the consequence of this term in the case of ${ }^{4} \mathrm{He}$ where the interface is very mobile. The dispersion relation for crystallization waves becomes linear : $\omega=c_{0} k$. The phonon transmission through the interface is enhanced. The Kapitza resistance is thus decreased, but presents always the anomalous dependence $R_{\mathrm{K}} \propto T^{-5}$ predicted by Marchenko and Parshin on other grounds.
\end{abstract}

1. Introduction. - Melting of ${ }^{4} \mathrm{He}$ crystals is a very fast process. It means that a very small difference between the chemical potentials in the liquid and the solid phases results in high velocities of the interface. This is due to the progressive disappearance at low temperature of any excitation in both the superfluid and the solid h.c.p. phases [1]. This quantitative difference with ordinary materials has strong qualitative effects. The existence of crystallization waves at the interface has been discussed [1] and experimentally proved [2]. The sound transmission is also affected [3]. Suppose that a sound wave is emitted in the liquid : because of its high mobility the interface almost maintains a constant pressure and nearly no stress is transmitted to the solid. The transmission of sound has been observed [4] to be far less than it would be, only due to the difference in acoustic impedances, without melting. The consequence of this reduction in the transmission of sound is an enhancement of the Kapitza resistance $R_{\mathrm{K}}$ of the interface beyond its ordinary $T^{-3}$ temperature dependence. This has been discussed by Marchenko and Parshin [5]. The liquid-solid surface tension makes the transmission finite, and frequency dependent. This results in a $T^{-5}$. dependence which has been experimentally observed [6,7].

The purpose of this letter is to point out that, at high frequencies, another property of the interface becomes important. One must take into account the fact that at the melting a strong structural 
rearrangement occurs. This results in a supplementary surface energy, a surface "kinetic" energy, proportional to $J^{2}$, where $J$ is the mass flux across the interface.

The paper is organized as follows. First we give a qualitative interpretation of the origin and the influence of this « kinetic » energy. Second we examine how the ordinary equations are modified by its introduction. Then, with a simplified model for the solid, we calculate both the dispersion equation for the crystallization waves, and the transmission coefficient for sound waves. Finally we compare with the available experimental results.

2. Qualitative interpretation. - The surface energy between solid and liquid is known [8] to be mainly due to the structural misfit between these two phases. In order to make a crystal from the liquid, one must transform the short range order into long range order. It may occur by small displacements of the atoms, like reorientation of small clusters.

The crystallization of some volume of liquid takes a finite time, the time it takes to the interface to go through this volume. To these atomic displacements corresponds then a supplementary kinetic energy. This kinetic energy occurs only if there is growing or melting of the crystal. It is then proportional to $J^{2}$.

In order to have a symmetric expression we shall write it $\left(\rho_{\mathrm{S}}\right.$ and $\rho_{\mathrm{L}}$ are the densities of the solid and the liquid) :

$$
\frac{\sigma}{\rho_{\mathrm{S}} \rho_{\mathrm{L}}} \frac{J^{2}}{2} \text { per unit area of the interface. }
$$

We interpret then $\sigma$ as being the interface mass per unit area. An order of magnitude (probably an overestimation) is given by $: \rho_{\mathrm{S}} d$, where $d$ is the interatomic distance in the solid.

This mass will act as a self inductance in an electric circuit. In order to accelerate the rate of crystallization, one must enhance the difference in chemical potentials between the liquid and solid phases. This difference will be proportional to $\partial J / \partial t$.

The consequence is clear. At high frequency the melting and crystallization processes are blocked. The phonon transmission is controlled by the differences in acoustic impedances. At low frequency these processes are restored and the phonon transmission is small. Thus at low temperature, anomalous behaviour will occur for the Kapitza resistance.

3. Equations. - We restrict ourselves to a rough interface at zero temperature. The reference plane for the interface is taken as the $(\hat{x}, \hat{y})$ plane, the third or $z$ direction being oriented toward the liquid phase (Fig. 1). We assume translational invariance in the $\hat{y}$ direction. We do not include the crystalline anisotropy in the $(\hat{x}, \hat{y})$ plane, which would complicate the equations without supporting new physical ideas.

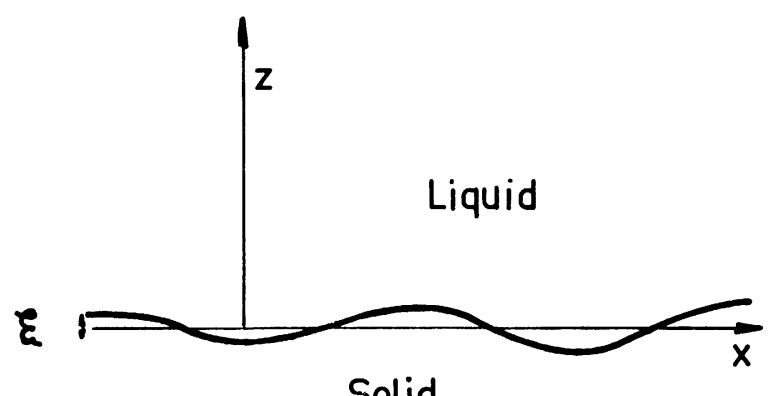

Solid

Fig. 1. - Notations used in the problem of crystallization waves. 
The position of the interface is given by the small amplitude $\xi(x, t)$. The first equation is the mass conservation :

$$
\rho_{\mathrm{S}}\left(u_{\mathrm{S}}-u\right)=\rho_{\mathrm{L}}\left(u_{\mathrm{L}}-u\right)=J
$$

where $u=\partial \xi / \partial t ; u_{\mathrm{S}}$ and $u_{\mathrm{L}}$ are the $z$ components of the liquid and solid phase velocities. We shall now write and discuss the energy conservation :

$$
\begin{aligned}
& \tilde{\alpha} \frac{\partial}{\partial x}\left(\frac{\partial \xi}{\partial x} \frac{\partial \xi}{\partial t}\right)+\left(P_{\mathrm{S}} u_{\mathrm{S}}-P_{\mathrm{L}} u_{\mathrm{L}}\right)+\left(\rho_{\mathrm{S}} e_{\mathrm{S}} u_{\mathrm{S}}-\rho_{\mathrm{L}} e_{\mathrm{L}} u_{\mathrm{L}}\right)= \\
&=\frac{\partial}{\partial t}\left[\frac{1}{2}\left(\alpha+\frac{\mathrm{d}^{2} \alpha}{\mathrm{d} \phi^{2}}\right)\left(\frac{\partial \xi}{\partial x}\right)^{2}+\frac{\sigma}{\rho_{\mathrm{S}} \rho_{\mathrm{L}}} \frac{J^{2}}{2}\right]
\end{aligned}
$$

$e_{i}$ is the internal energy per unit mass of the phase $i$, and $P_{i}$ its pressure.

On the left hand side of the equation (2), the first term represents the work of tension forces at the interface :

$$
\tilde{\alpha}=\alpha+\frac{\mathrm{d}^{2} \alpha}{\mathrm{d} \phi^{2}}
$$

where $\alpha$ is the surface energy, and $\phi=\partial \xi / \partial x$ the angle between the interface and the $\hat{x}$ direction.

The second term represents the work of pressure forces, and the third term is the difference between the incoming and out going energy fluxes. Note that we have neglected higher order terms like $\left|\rho_{\mathrm{S}}\left(u_{\mathrm{L}}^{2} / 2\right) u_{\mathrm{S}}\right|$ in these fluxes. We recall that we have neglected also the latent heat, heat fluxes and all dissipative processes. In this approximation, for each phase, the chemical potential $\mu$ per unit mass is :

$$
\mu_{i}=e_{i}+\frac{P_{i}}{\rho_{i}}
$$

The right hand side of the equation (2) represents the change in the static and kinetic energy of the interface. In the static part, the first term

$$
\left[\frac{\alpha}{2}\left(\frac{\partial \xi}{\partial x}\right)^{2}\right]
$$

corresponds to the change in the interface surface and the second term

$$
\left[\frac{1}{2} \frac{\mathrm{d}^{2} \alpha}{\mathrm{d} \phi^{2}}\left(\frac{\partial \xi}{\partial x}\right)^{2}\right]
$$

to the dependence of $\alpha$ in the crystalline orientation.

We shall use the notation :

$$
P_{i}=P_{0}+p_{i}
$$

where $P_{0}$ is the equilibrium pressure. Note that $p_{\mathrm{S}}$ represents, in fact, the uniaxial stress : $-\sigma_{z z}$. Using equations (1) and (3), the energy conservation equation becomes :

$$
J\left(\mu_{\mathrm{S}}-\mu_{\mathrm{L}}\right)+\left(p_{\mathrm{S}}-p_{\mathrm{L}}+\tilde{\alpha} \frac{\partial^{2} \xi}{\partial x^{2}}\right) u=\frac{\sigma}{\rho_{\mathrm{S}} \rho_{\mathrm{L}}} J \frac{\partial J}{\partial t} .
$$


In order to ensure the Galilean invariance we thus must have :

$$
p_{\mathrm{S}}-p_{\mathrm{L}}+\tilde{\alpha} \frac{\partial^{2} \xi}{\partial x^{2}}=0
$$

which is the momentum conservation equation. The energy conservation reduces then to :

$$
\mu_{\mathrm{S}}-\mu_{\mathrm{L}}=\frac{p_{\mathrm{S}}}{\rho_{\mathrm{S}}}-\frac{p_{\mathrm{L}}}{\rho_{\mathrm{L}}}=\frac{\sigma}{\rho_{\mathrm{S}} \rho_{\mathrm{L}}} \frac{\partial J}{\partial t}
$$

It is important to note that $\sigma$ does not appear in the momentum conservation (Eq. 4). There is thus no "surface momentum " associated to this "kinetic " energy. This occurs because the surface kinetic energy is in fact Galilean invariant. Indeed $J$, being proportional to $\left(u_{\mathrm{L}}-u_{\mathrm{S}}\right)$, is itself Galilean invariant.

4. Crystallization waves. - Here we consider only waves whose phase velocity is much smaller than the elastic transverse waves velocity in the solid, which is itself smaller than the longitudinal sound velocity in both liquid and solid. Thus we can consider the liquid as incompressible and the solid as undeformed.

The derivation of the dispersion equation is then classical [9]. One can write the velocity $\mathbf{v}_{\mathbf{L}}$ of the liquid as : $\mathbf{v}_{\mathrm{L}}=-\operatorname{grad} \phi_{\mathrm{L}}$. Neglecting gravity :

$$
\rho_{\mathbf{L}} \frac{\partial \mathbf{v}_{\mathbf{L}}}{\partial t}=-\operatorname{grad} p_{\mathbf{L}}
$$

We thus can take $p_{\mathrm{L}}=\rho_{\mathrm{L}} \frac{\partial \phi_{\mathrm{L}}}{\partial t}$. With a harmonic form for $\phi_{\mathrm{L}}$ :

$$
\phi_{\mathrm{L}}=\phi(z) \mathrm{e}^{i k x_{\mathrm{e}}-i \omega t}
$$

the incompressibility imposes $: \phi(z)=\phi_{0} \mathrm{e}^{-k z}$.

In the solid frame

and

$$
J=\frac{\rho_{\mathrm{S}} \rho_{\mathrm{L}}}{\rho_{\mathrm{S}}-\rho_{\mathrm{L}}} u_{\mathrm{L}}=\frac{\rho_{\mathrm{S}} \rho_{\mathrm{L}}}{\rho_{\mathrm{S}}-\rho_{\mathrm{L}}} k \phi_{\mathrm{L}}
$$

$$
u=\frac{\partial \xi}{\partial t}=-i \omega \xi=-\frac{\rho_{\mathrm{L}} u_{\mathrm{L}}}{\rho_{\mathrm{S}}-\rho_{\mathrm{L}}}=-\frac{\rho_{\mathrm{L}} k \phi_{\mathrm{L}}}{\rho_{\mathrm{S}}-\rho_{\mathrm{L}}} .
$$

Using (4) and (5) one finally obtains the dispersion relation [10] :

$$
\omega^{2}=\frac{\left(\tilde{\alpha} \rho_{\mathrm{L}} /\left(\rho_{\mathrm{S}}-\rho_{\mathrm{L}}\right)^{2}\right) k^{3}}{1+\left(\sigma \rho_{\mathrm{S}} /\left(\rho_{\mathrm{S}}-\rho_{\mathrm{L}}\right)^{2}\right) k} .
$$

At low frequency we recover the usual result $\omega \propto k^{3 / 2}$. However at high frequency, we obtain a linear dispersion $\omega=c_{0} k$ with

$$
c_{0}^{2}=\frac{\tilde{\alpha} \rho_{\mathrm{L}}}{\sigma \rho_{\mathrm{S}}}
$$


This can be easily understood. At low frequency the inertia comes from the liquid, whose movement occurs on a depth $k^{-1}$. This gives the extra factor $k$ in $\omega^{2}$. At high frequency however, the main inertia comes from the interface and does not depend on frequency. Thus we recover the linear dispersion.

Let us obtain an order of magnitude for $c_{0}$; putting $\sigma=\rho_{\mathrm{S}} d$. With $d \cong 3 \AA$ and $\tilde{\alpha} \cong 2 \times 10^{-4} \mathrm{~J} / \mathrm{m}^{2}$ [11] we obtain :

$$
c_{0} \cong 60 \mathrm{~m} / \mathrm{s}
$$

which is far less than the transverse sound velocity of the solid. This justifies our approximation.

The most important consequence is that the crystallization waves are not damped at high frequency by emission of transverse sound. They can be observed at any frequency.

As has been already pointed out [5], this mode is different from the Rayleigh wave which occurs anyway at the interface due to the elasticity of the solid. If the real value of $\sigma$ is much smaller than our rough estimation a strong coupling between these two modes will occur : in the incompressible case (where $c_{\mathrm{L}}$ and $c_{\mathrm{S}}$ are much larger than the transverse sound velocity in the solid : $c_{\mathrm{t}}$ ), the dispersion relation for the surface waves becomes

$$
\left(\rho_{\mathrm{S}}-\rho_{\mathrm{L}}\right)+\dot{k}\left\{\rho_{\mathrm{S}}\left(l_{\sigma}+\frac{v^{2} l_{\alpha}}{s^{2}}\right)-\rho_{\mathrm{L}}\left(\frac{l_{\alpha}}{v^{2}}+\frac{l_{\sigma} v^{4}}{s^{2}}\right)\right\}+\frac{l_{\sigma} l_{\alpha}}{s^{2}}\left(\rho_{\mathrm{S}}-\rho_{\mathrm{L}}\right) k^{2}=0
$$

where

and

$$
v=\frac{\omega}{c_{\mathrm{t}} k} ; \quad l_{\sigma}=\frac{\sigma}{\rho_{\mathrm{S}}-\rho_{\mathrm{L}}} ; \quad l_{\alpha}=\frac{\tilde{\alpha}}{\left(\rho_{\mathrm{S}}-\rho_{\mathrm{L}}\right) c_{\mathrm{t}}^{2}}
$$

$$
s^{2}=4\left\{\left(1-v^{2}\right)^{1 / 2}-\left(1-\frac{v^{2}}{2}\right)^{2}\right\}
$$

Note the ratio of the two characteristic lengths $: l_{\alpha} / l_{\sigma}=c_{0}^{2} \rho_{\mathrm{S}} / c_{\mathrm{t}}^{2} \rho_{\mathrm{L}}$. On the figure 2 , we have plotted $v^{2}$ versus $k l_{\alpha}$, in the two cases $l_{\sigma}=10 l_{\alpha}$ (small coupling) and $l_{\sigma}=0$ (maximum coupling).

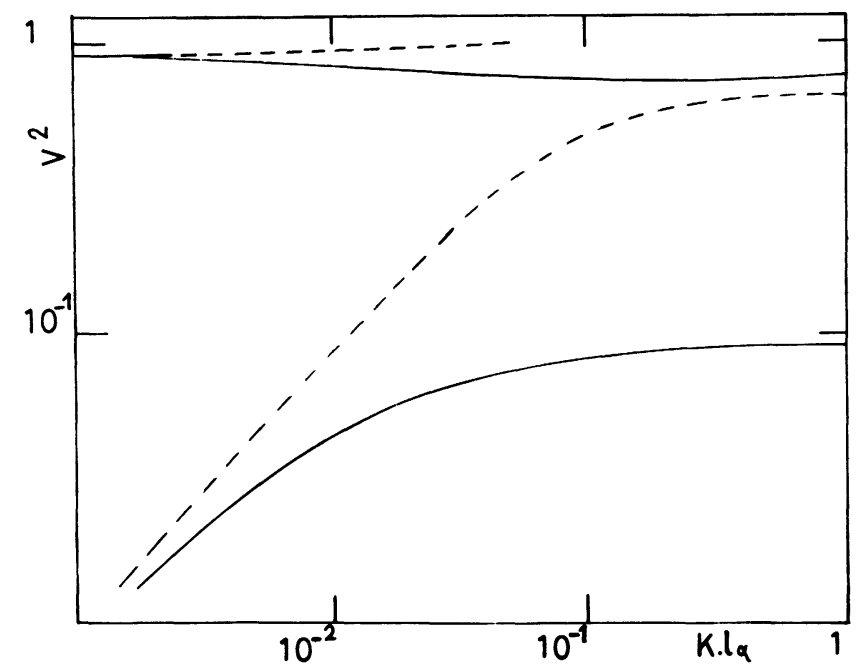

Fig. 2. - Dispersion relation for the two surface modes in two different cases : - $l_{\sigma}=10 l_{\alpha}$ (large surface mass); ------- $l_{\sigma}=0$ (no surface mass). For each case, the upper curve corresponds to the Rayleigh wave, and the lower curve to the crystallization wave. 
In this $\log -\log$ plot, the usual capillary dispersion relation $\omega^{2} \propto k^{3}$ has a slope 1 . The order of magnitude of $l_{\alpha}$ is $2 \AA$. Thus for $k l_{\alpha} \gtrsim 1$ we could have taken into account the roton and zone boundary dispersions.

5. Transmission of sound. - In this section, in order to demonstrate the effect of the surface inertia and to stress the difference with the Marchenko-Parshin effect, we shall calculate the sound transmission coefficient. In all cases we shall consider only the longitudinal mode in the solid.

We thus consider an incident sound wave from the liquid with a pressure amplitude $p_{0}$, whose wave vector $\mathbf{k}_{\mathbf{i}}$ makes the angle $\theta_{\mathbf{L}}$ with the normal to the surface. The transmitted wave has a pressure amplitude $\tau p_{0}$ and a wave vector $\mathbf{k}_{\mathbf{t}}$ which makes the angle $\theta_{\mathbf{s}}$ with the normal. The pressure amplitude of the reflected wave is $R p_{0}$. The normal components of the velocities in the liquid and the solid phases are :

$$
\begin{aligned}
& u_{\mathrm{L}}=-\frac{(1-R) p_{0}}{\rho_{\mathrm{L}} c_{\mathrm{L}}} \cos \theta_{\mathrm{L}} \\
& u_{\mathrm{S}}=-\frac{\tau p_{0}}{\rho_{\mathrm{S}} c_{\mathrm{S}}} \cos \theta_{\mathrm{S}}
\end{aligned}
$$

where $c_{\mathbf{L}}$ and $c_{\mathbf{S}}$ are the velocities of longitudinal sound in the liquid and solid phases. Using the relation

$$
J=\frac{\rho_{\mathrm{L}} \rho_{\mathrm{S}}}{\rho_{\mathrm{S}}-\rho_{\mathrm{L}}}\left(u_{\mathrm{L}}-u_{\mathrm{S}}\right)
$$

the equation (5) gives :

$$
\frac{(1+R)}{\rho_{\mathrm{L}}}-\frac{\tau}{\rho_{\mathrm{S}}}=-\frac{i \omega \sigma}{\rho_{\mathrm{S}}-\rho_{\mathrm{L}}}\left\{\frac{(1-R) \cos \theta_{\mathrm{L}}}{\rho_{\mathrm{L}} c_{\mathrm{L}}}-\frac{\tau \cos \theta_{\mathrm{S}}}{\rho_{\mathrm{S}} c_{\mathrm{S}}}\right\} .
$$

As for equation (4), if we remark that :

$$
k_{\mathrm{i}_{x}}=\frac{\omega}{c_{\mathrm{L}}} \sin \theta_{\mathrm{L}}=\frac{\omega \sin \theta_{\mathrm{S}}}{c_{\mathrm{S}}}
$$

it gives :

$$
(1+R)-\tau=-i \omega \frac{\tilde{\alpha} \sin \theta_{\mathrm{S}} \sin \theta_{\mathrm{L}}}{c_{\mathrm{S}} c_{\mathrm{L}}\left(\rho_{\mathrm{S}}-\rho_{\mathrm{L}}\right)}\left\{\frac{(1-R) \cos \theta_{\mathrm{L}}}{c_{\mathrm{L}}}-\frac{\tau \cos \theta_{\mathrm{S}}}{c_{\mathrm{S}}}\right\} .
$$

In order to obtain a more compact form for these two equations we shall use the notations :

$$
\begin{gathered}
\frac{\cos \theta_{\mathrm{L}}}{c_{\mathrm{L}}}=\lambda_{\mathrm{L}} ; \quad \frac{\cos \theta_{\mathrm{S}}}{c_{\mathrm{S}}}=\lambda_{\mathrm{S}} \\
\frac{\tilde{\alpha} \sin \theta_{\mathrm{S}} \sin \theta_{\mathrm{L}}}{c_{\mathrm{S}} c_{\mathrm{L}}\left(\rho_{\mathrm{S}}-\rho_{\mathrm{L}}\right)}=A ; \quad \frac{\sigma}{\rho_{\mathrm{S}}-\rho_{\mathrm{L}}}=B .
\end{gathered}
$$

Then :

$$
\begin{gathered}
(1+R)-\tau=-i A \omega\left\{(1-R) \lambda_{\mathrm{L}}-\tau \lambda_{\mathrm{S}}\right\} \\
(1+R)-\frac{\rho_{\mathrm{L}}}{\rho_{\mathrm{S}}} \tau=-i B \omega\left\{(1-R) \lambda_{\mathrm{L}}-\frac{\rho_{\mathrm{L}}}{\rho_{\mathrm{S}}} \tau \lambda_{\mathrm{S}}\right\} .
\end{gathered}
$$


If we make the simultaneous substitutions $\tau \leftrightarrow \tau^{\prime}=\frac{\rho_{\mathrm{L}}}{\rho_{\mathrm{S}}} \tau, \rho_{\mathrm{S}} \leftrightarrow \rho_{\mathrm{L}}, A \leftrightarrow B$ we obtain similar equations. This shows the symmetry between surface tension and mass effects. The solution is :

$$
\tau=\frac{-2 i(B-A) \rho_{\mathrm{S}} \lambda_{\mathrm{L}} \omega}{\left(\rho_{\mathrm{S}}-\rho_{\mathrm{L}}\right)+i \omega\left\{A\left(\rho_{\mathrm{L}} \lambda_{\mathrm{L}}+\rho_{\mathrm{S}} \lambda_{\mathrm{S}}\right)-B\left(\rho_{\mathrm{S}} \lambda_{\mathrm{L}}+\rho_{\mathrm{L}} \lambda_{\mathrm{S}}\right)\right\}+\omega^{2} A B \lambda_{\mathrm{S}} \lambda_{\mathrm{L}}\left(\rho_{\mathrm{S}}-\rho_{\mathrm{L}}\right)} .
$$

Remember that it corresponds to the pressure amplitude transmission. The energy transmission is then :

$$
T_{\mathrm{E}}=\frac{4(B-A)^{2} \rho_{\mathrm{S}} \rho_{\mathrm{L}} \lambda_{\mathrm{L}} \lambda_{\mathrm{S}} \omega^{2} /\left(\rho_{\mathrm{S}}-\rho_{\mathrm{L}}\right)^{2}}{\left(1+\omega^{2} A B \lambda_{\mathrm{S}} \lambda_{\mathrm{L}}\right)^{2}+\frac{\omega^{2}}{\left(\rho_{\mathrm{S}}-\rho_{\mathrm{L}}\right)^{2}}\left\{A\left(\rho_{\mathrm{L}} \lambda_{\mathrm{L}}+\rho_{\mathrm{S}} \lambda_{\mathrm{S}}\right)-B\left(\rho_{\mathrm{S}} \lambda_{\mathrm{L}}+\rho_{\mathrm{L}} \lambda_{\mathrm{S}}\right)\right\}^{2}} .
$$

6. Discussion. - We now turn to the orders of magnitude.

In the expression of $T_{\mathrm{E}}$ the term $\omega^{2} A B \lambda_{\mathrm{L}} \lambda_{\mathrm{S}}$ which appears in the denominator is much smaller than 1 , except for frequencies larger than the roton frequencies (taking a reasonable value for $\sigma$ )! We can thus neglect it.

Let us now compare $A$ and $B$. Their equality corresponds roughly to

$$
c_{0}^{2}=c_{\mathrm{S}} c_{\mathrm{L}}
$$

We expect $c_{0}$ to be much smaller, thus $B \gg A$. In any case, using our expression for $T_{\mathrm{E}}$ we can calculate the Kapitza resistance and compare it with recent experiments [6,7]. This point will be developed later and we give here only the result. In the low temperature regime, most of the crystallographic orientations give [7] :

$$
R_{\mathrm{K}} T^{5} \cong 2 \times 10^{-2} \frac{\mathrm{cm}^{2} \mathrm{~K}^{6}}{\mathrm{~W}}
$$

However, taking $\sigma=0(B=0)$ we find [12] :

$$
R_{\mathrm{K}} T^{5} \cong 1.3 \frac{\mathrm{cm}^{2} \mathrm{~K}^{6}}{\mathrm{~W}}
$$

Thus the transmission cannot be only due to the surface tension and $B \gg A$.

Taking $A=0$, comparing with the experimental result gives :

$$
\sigma \cong 0.24 \times 10^{-8} \mathrm{~kg} / \mathrm{m}^{2} \cong \frac{\rho_{\mathrm{S}} d}{25}
$$

If this value is correct it is necessary to take into account the coupling between crystallization and Rayleigh waves in the determination of their dispersion relations.

7. Conclusion. - In this paper we have discussed the influence of the "kinetic " energy of the liquid-solid interface of helium.

It results in a linear dispersion for capillary waves at high frequency. In this limit they are thus probably not damped by emission of bulk sound waves.

As for the Kapitza resistance, it gives the same anomalous behaviour $R_{\mathrm{K}} \propto T^{-5}$ as the capillary effect discussed by Marchenko and Parshin. However the larger transmission it allows is 
necessary to interpret the experimental results. The Kapitza resistance is in fact mainly due to this interface inertia.

Note. - D. O. Edwards has drawn our attention on the fact that the surface of separation may be defined in different ways [13]. If one attributes no atoms to the surface, one defines the " dividing surface ". The energy per unit area of this surface depends on its curvature. If we want, following Gibbs, to have a surface energy proportional to its area, we must take̊ another surface : the " surface of tension". This introduces a "surface mass " $\Gamma$ which in our case is $\Gamma=\left(\rho_{\mathrm{S}}-\rho_{\mathrm{L}}\right) \delta$, where $\delta$ is the distance between these two surfaces.

Our mass $\sigma$ does not correspond to a dependence on the curvature of the specific energy of the " dividing surface ", but to its dependence on $J$. Moreover, attributing all the acoustic transmission to $\Gamma$ leads to a large value of $\delta$ for a flat surface : $\delta=\delta_{0}=1.5 \AA$, while one would expect a $\delta_{0}$ very small : $\delta_{0} \cong 0.1 \AA$ for the liquid-gas interface.

Acknowledgments. - We are indebted to P. Nozières, P. Averbuch, M. Papoular and the whole helium group in CRTBT for critically discussing the manuscript. We would also thank D. O. Edwards for a very fruitful and lively discussion.

\section{References}

[1] Andreev, A. and Parshin, A., Sov. Phys. J.E.T.P. 48 (1979) 763.

[2] Keshishev, K. O., Parshin, A. and Babkin, A., Sov. Phys. J.E.T.P. 53(1981) 362.

[3] Castaing, B. and Nozières, P., J. Physique 41 (1980) 701.

[4] Castaing, B., Balibar, S. and Laroche, C., J. Physique 41 (1980) 897.

[5] Marchenko, V. and Parshin, A., J.E.T.P. Lett. 31 (1980) 724.

[6] Huber, T. E. and Maris, H. J., Phys. Rev. Lett. 47 (1981) 1907.

[7] Puech, L., HÉbral, B., Thoulouze, D. and Castaing, B., to be published.

[8] Bonnissent, A., Gauthier, E. and Finney, J. L., Philos. Mag. B 39 (1979) 49.

[9] Landau, L. and LifChitz, E., Cours de Physique Théorique (Ed. Mir) Tome VI, § 61.

[10] Kosevitch, A. M. and Kosevitch, Yu. A., L.T. 16, Physica 108B + C (1981) 1195 have found a similar equation, examining the influence of "condensed steps" on a rough interface. We think that it is not adequate to use classical results on steps in such conditions.

[11] Balibar, S., Edwards, D. O. and Laroche, C., Phys. Rev. Lett. 42 (1979) 782.

[12] Maris, H. J. and Huber, T. E. (to be published), by numerical integration, including the transverse phonon contribution, have found a much smaller value in this case : $R_{\mathrm{K}} T^{5}=0.1 \frac{\mathrm{cm}^{2} \mathrm{~K}^{6}}{\mathrm{~W}}$ (which anyway does not modify our conclusion). This is probably due to the fact that the Marchenko and Parshin coupling is much stronger close to $\theta_{\mathrm{L}}=\pi / 2$; in this limit only the transverse waves are coupled to the liquid. Such an effect does not occur for the coupling by inertia and the value of $\sigma$ we deduce from our calculation would be a good approximation.

[13] GibBS, J. W., Scientific papers (Dover Publications, New York).

Andreev, A. and Kompaneets, D., Sov. Phys. J.E.T.P. 34 (1972) 1316.

Edwards, D. O., Eckardt, J. R. and Gasparini, F. M., Phys. Rev. A 9 (1974) 2070. 